\title{
Grain-scale DEM study of open-ended pipe pile penetration in granular soils
}

\author{
Michail Komodromos ${ }^{1, *}$, Gaël Combe ${ }^{1, * *}$, and Gioacchino Viggiani ${ }^{1, * * *}$ \\ ${ }^{1}$ Univ. Grenoble Alpes, Grenoble INP, CNRS, 3SR, F-38000, Grenoble, France
}

\begin{abstract}
Open-Ended Pipe Piles (OEPP) are particularly popular in offshore engineering. An important feature of the installation of these piles is the rate with which soil enters the pile from the bottom, and its interaction with the internal pile shaft. The response of OEPP crucially depends on the occurrence of soil plugging, which can make the behavior of an OEPP similar to a pile of solid cross section. Plugging is generally attributed to arching effects in the soil; therefore, understanding this phenomenon requires an investigation at the grain scale. This is precisely the objective of this study, where the Distinct Element Method (DEM) is used to study the installation of an Open-Ended Pipe Pile in a Virtual Calibration Chamber comprising 128000 grains, under constant horizontal stress. Despite the relatively small number of particles, this numerical model is found to be able to reproduce several aspects of the mechanisms actually observed in the field. The results are compared to those obtained from actual experiments of miniature pile penetration tests. Stress and strain fields that develop in the soil inside and outside the pile provide interesting data and shed light on the mechanisms at play during OEPP installation, especially as for the influence of grains interlocking.
\end{abstract}

\section{Introduction}

The penetration of hollow-sectioned pipe piles, usually referred to as Open-Ended Pipe Piles (OEPP), is associated with complex interaction between the soil and the pile. A major aspect of such interaction is the granular flow inside the pile, which can either occur unrestrained or result into the formation of a soil plug. By definition, plugging is the state when the soil-pile shear stress along the internal shaft increases to the point where the entering of additional soil is partially or fully prevented during further embedding of the pile. The macroscopic effect of plugging is a difference between the internal soil column length and the embedding length, along with a much stiffer vertical response of the pile. It is generally suggested that plugging is due to arching effects in the soil. Therefore, its understanding calls for studies at the grain scale, similar to what was recently done experimentally for the installation of conventional, closed-ended piles using $\mathrm{x}$-ray micro tomography and image analysis [1-4]. In the present study, a grainscale analysis of the installation of Open-Ended Pipe Piles penetration is carried out numerically, using the Discrete Element Method.

\section{Numerical pile penetration: the DEM model}

The numerical experiment consists in driving a $1 \mathrm{~cm}$ diameter hollow cylinder with a thickness of $1 \mathrm{~mm}$ into a granular material made of 128000 rigid spheres of diameters homogeneously distributed between $0.95 \mathrm{~mm}$ to $1 \mathrm{~mm}$, see

\footnotetext{
*e-mail: michail.komodromos@tuhh.de

**e-mail: gael.combe@grenoble-inp.fr

***e-mail: cino.viggiani@3sr-grenoble.fr
}

A video is available at https://doi.org/10.48448/ckhp-qm55
Fig. 1. The granular assembly is confined by rigid walls with an applied normal stress of $100 \mathrm{kPa}$. During pile installation, the top and the bottom walls are fixed, while the four lateral walls impose a constant normal stress of 100 $\mathrm{kPa}$, at all times under stiffness level of 1000 and damping factor of 0.05 . The pile moves downward at constant velocity, and eventually crosses the bottom of the box (the interaction between the pile and the top and bottom walls of the box is not taken into account).

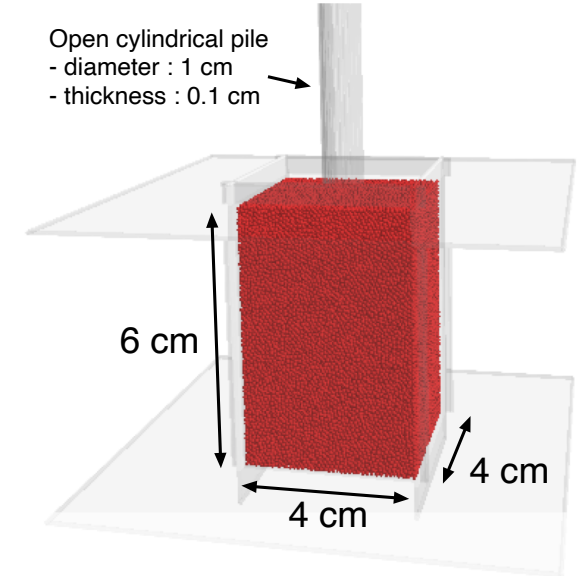

Figure 1. Testing Configuration.

As in any DEM model, when the particles are in contact, a normal elastic and a tangential incremental elasto-plastic force are computed $[5,6]$. When perfectly spherical frictional particles are in contact, rolling without sliding yields unrealistically low shear resistance of the granular assembly. This can be avoided by using particles with complex shapes (e.g., clumps, polygons, ... [7]), or by adding 
another contact law that drastically reduces rolling without sliding: a resisting moment that introduces a contact length $[6,8]$. This rolling resistance can be seen as a way to mimic complex particle shapes [9]. The contact rolling resistance can be used for particle-to-particle interaction but also at the contact of spheres with the pile shaft. In order to study the effect of rolling resistance on the overall response of the system, various situations are analyzed in this study, where rolling resistance was set by standard contact length of $0.1 \mathrm{~mm}$ : no rolling resistance $(\boldsymbol{F R})$, rolling resistance at the particle-to-particle contacts only $(-\boldsymbol{R})$, and rolling resistance for all contacts, including contacts between the particles and the pile $(+\boldsymbol{R})$.

In order to evaluate the shear resistance of the granular assembly, some vertical compression tests with constant lateral stress in quasi-static regimes (inertial number $I \leq 10^{-3}$ ) are performed. All samples show the typical response of dense sands, characterized by a peak deviator stress and dilatancy. The obtained values of friction and dilatancy angles, with and without rolling resistance, are given in Table 1.

\begin{tabular}{|l|c|c|}
\hline Samples & FR & -R / +R \\
\hline Friction angle $\left(^{\circ}\right)$ & 31.6 & 40.3 \\
Dilatancy angle $\left(^{\circ}\right)$ & 11.5 & 15.6 \\
\hline \hline
\end{tabular}

Table 1. Friction and dilatancy angles of the granular assemblies, as determined from numerical triaxial compression tests.

The pile installation was performed in driving velocity respecting a standard value of inertia number equal to $5 * 10^{-5}$. Fig. 2 shows the evolution of the total vertical load acting on the pile and the load at the pile tip during installation over $0.06 \mathrm{~m}$, until the pile tip reaches the bottom of the granular box. Results from both simulations with and without rolling resistance are plotted. One can observe that the tip load is constant, while the total load increases with the increase of the contact surface between the pile shaft and the granular assembly. This result is consistent with what is classically observed in the installation of real piles. Moreover, one can observe that the total and the tip loads strongly depend on the shear strength of the granular assembly. The introduction of a rolling resistance at the particle-to-particle contacts increases more than twice the pile tip resistance, whereas the effect of a rolling resistance at the contact between particles and pile shaft ( $+R$ sample) is negligible.

\section{Kinematics}

The obtained kinematic fields are substantially axisymmetric - the axis of symmmetry being the axis of the pile. Therefore, a cylindrical coordinate system is used in the following, with displacements shown in the radial, orthoradial and vertical directions, $\left(u_{r}, u_{\theta}, u_{z}\right)$ respectively.

In Fig. 3, particles displacements are plotted in a single half-plane, with the vertical section of the pile represented as a white bar; particles to the left of this bar are inside the shaft. Note that, for the ease of the reader, only a few (one out of five) displacement vectors are shown in Fig. 3-b.

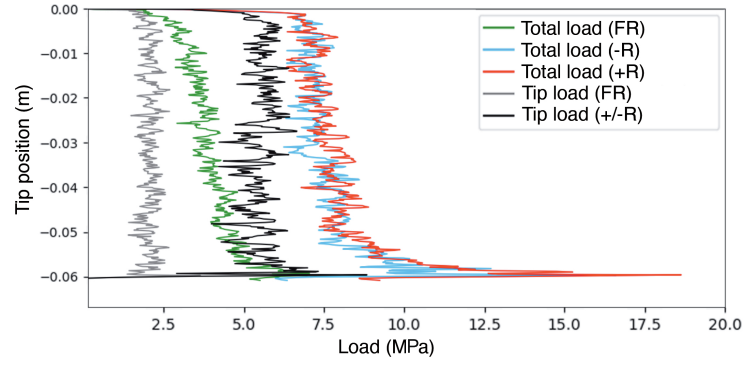

Figure 2. Macroscopic response to pile installation: total and pile tip load.

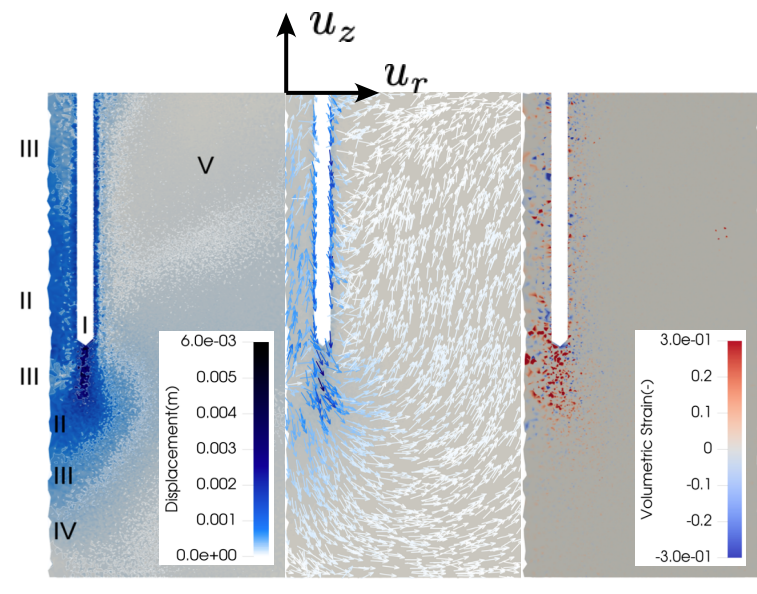

(a)

(b)

(c)

Figure 3. Kinematic fields in the granular assembly for a displacement increment of $8 \mathrm{~mm}$ of the pile (tip position moves from $u_{z}=-0.031 \mathrm{~m}$ to $u_{z}=-0.039 \mathrm{~m}$ ): (a) displacement amplitude, (b) displacement vectors, (c) volumetric strains.

Fig. 3-a shows the (norm of) displacement field in the box when the pile tip moves from $u_{z}=-0.031 \mathrm{~m}$ down to $u_{z}=-0.039 \mathrm{~m}$. Light blue means zero displacement, while darkest blue corresponds to a displacement of $6 \mathrm{~mm}$. Five regions can be identified, from $I$ to $V$, depending on the displacement amplitude of the particles: in region $I$ the displacements are as large as the displacement of the pile, while in region $V$ the displacements are close to zero. Fig. 3-b shows the direction of the displacement, with the color still representing its amplitude (with the same color scale as in Fig. 3-a). Far from the pile (for $u_{r}>1.5 \mathrm{~mm}$ ), particle displacements are rather small (zone $V$ ). Particles located under the pile tip move down, whereas those inside the pile move up with an amplitude of around $2 \mathrm{~mm}$. In both Figs. 3-a and 3-b, one can observe that an arch is formed from the base of the pile, with a displacement bulb moving downward. It can be seen that the particles in contact with the shaft of the pile move downwards, but with a smaller amplitude than the pile. This relative displacement between the pile and the particles implies a shear mechanism at the interface of the pile, both on the inner 
and outer side of the shaft. Based on the particle displacements (and a suitable triangulation of the particles), a strain tensor can be computed on (constant strain) tetrahedra. Fig. 3-c shows the volumetric strain field, clearly indicating volumetric expansion near the pile tip (red zone). Inside the pile, no particular trend is observed: some regions contract, while others dilate. A similar observation can be made in the sheared zone on the external side of the pile shaft. Interestingly, similar observations on OEPP driven into granular soil were reported in [10], based on experiments with $\mathrm{x}$-ray tomography.

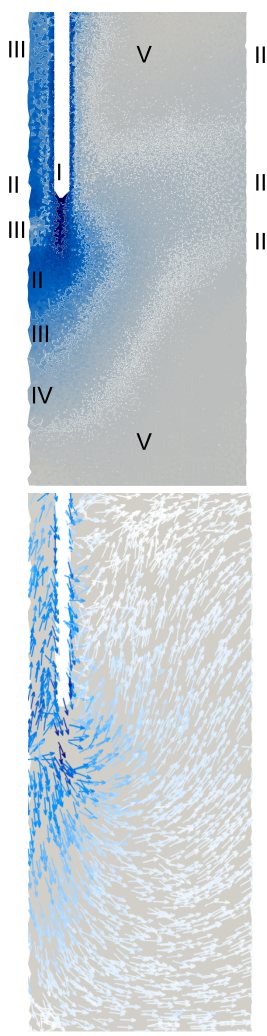

(a)

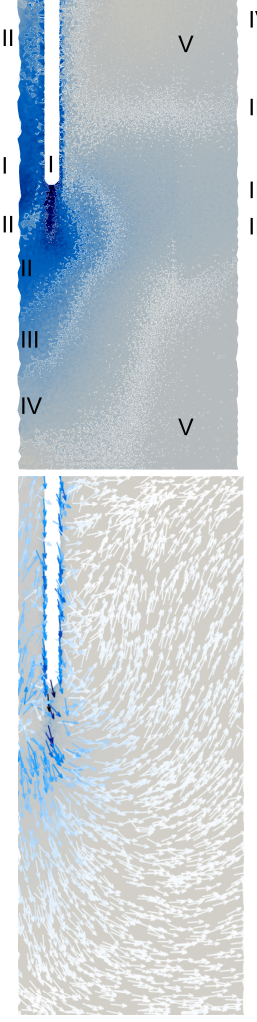

(b)

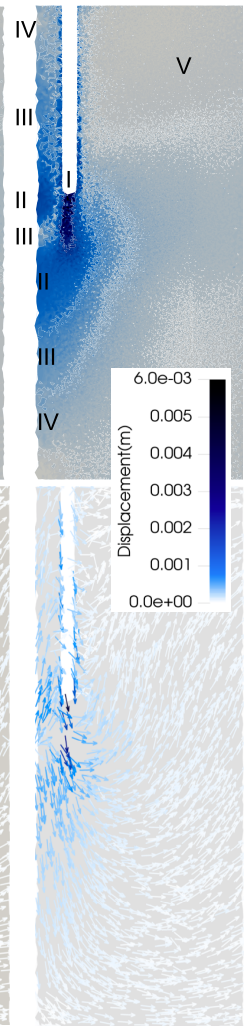

(c)
Figure 4. Kinematic fields for a displacement increment of $8 \mathrm{~mm}$ of the pile (tip position moves from $0.031 \mathrm{~m}$ to $0.039 \mathrm{~m}$ ); (a) $F R$, (b) $-R$, (c) $+R$.

Rolling resistance is known to ease arching [11]. Fig. 4 shows that in the absence of rolling resistance (FR sample, Fig. 4-a ), the displacement bulb (zones $I I$ and $I I I$ at the tip of the pile) appears to be less spread than when rolling resistance is taken into account (Figs. 4-b and 4-c). Other differences can be observed between the three cases in Fig. 4, but no clear conclusions can be drawn as for the effect of rolling resistance on particles kinematics.

\section{Stresses}

Contact forces between particles can be used to evaluate stresses at the particle scale, by means of the well-known Weber expression [12]. As for the displacements, stress is expressed using a cylindrical coordinate system: radial stress $\sigma_{r r}$, vertical stress $\sigma_{z z}$, and orthoradial stress $\sigma_{\theta \theta}$.
In the initial state (i.e., before pile installation), the stress is isotropic and homogeneous, with $\sigma_{r r}=\sigma_{\theta \theta}=\sigma_{z z}=$ $100 \mathrm{kPa}$ for all values of $r, \theta$, and $z$.

Fig. 5 shows the $\sigma_{z z}$ field for the three cases considered in this study $(F R,-R$, and $+R)$, when the pile tip is at a depth of $z=-0.039 \mathrm{~m}$. As expected, a concentration of vertical stress is observed at the pile tip, with higher values in the presence of rolling resistance (Figs. 5-b and $5-c)$. This is consistent with the fact that the tip resistance is twice higher for $-R$ and $+R$ samples, as it was shown in Fig. 2.

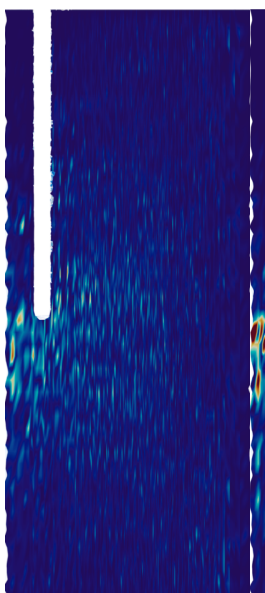

(a)

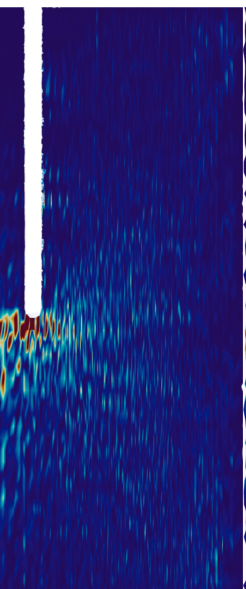

(b)

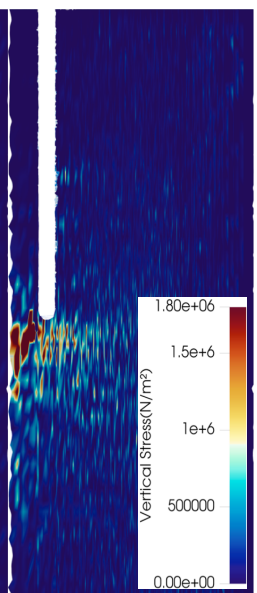

(c)
Figure 5. Distribution of vertical stress $\sigma_{z z}$ when the pile tip is at a depth of $z=-0.039 \mathrm{~m}$ : (a) $F R$, (b) $-R$, (c) $+R$.

While the stress on the boundaries of the box is maintained at $100 \mathrm{kPa}$ during pile installation, the stresses are no longer homogeneously distributed in the sample. Fig. 6 shows the vertical profiles of horizontally-averaged radial and orthoradial stress when the pile tip is at $z=$ $-0.039 \mathrm{~m}$. Both stress components (which are significantly affected by the presence of rolling resistance) are larger than $100 \mathrm{kPa}$ below the pile tip, and less than $100 \mathrm{kPa}$ above. It is worth noting that the average value of both stress components over the whole height of the sample (from $z=0 \mathrm{~m}$ to $z=-0.06 \mathrm{~m}$ ) is close to $100 \mathrm{kPa}$. It is clear that the high values of $\sigma_{\theta \theta}$ below the pile tip are also due to the boundary conditions used here (rigid walls); however, this trend in agreement with experimental measurements for closed pile [13, 14].

In order to better understand the response to pile penetration, let us focus now on the distribution of radial stress, $\sigma_{r r}$, on the inner and outer sides of the pile shaft, i.e., at $r=0.009 \mathrm{~m}$ and $r=0.01 \mathrm{~m}$, respectively. Once again, the effect of rolling resistance is also investigated, by comparing $F R$ and $+R$ samples. Fig. 7 shows that $\sigma_{r r}$ acting on the inner side of pile shaft is close to zero from $z=0 \mathrm{~m}$ to $z=-0.025 \mathrm{~m}$ and then significantly increases from $z=-0.025 \mathrm{~m}$ to the pile tip $(z=-0.039 \mathrm{~m})$ for $F R$ sample. However, in the presence of rolling resistance $(+R$ sample), the radial stress is close to zero all along the inner shaft. Even if particles entered the pile during its penetration, the arching that develops at the pile tip (Fig. 5-c) 


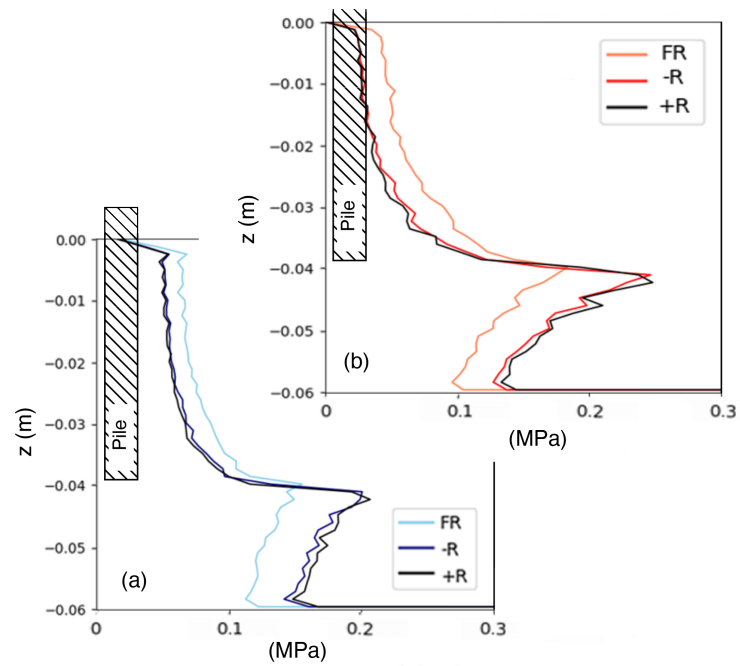

Figure 6. Horizontally-averaged stress profiles: (a) $\sigma_{r r}$ (b) $\sigma_{\theta \theta}$.

avoids stress transfer from the particles at the pile tip to the particles inside the pile. Fig. 7 also shows that on the outer side of the pile shaft, the normal stress increases dramatically at the tip when the granular assembly is characterized by high values of friction and dilatancy angles $(+R$ sample).

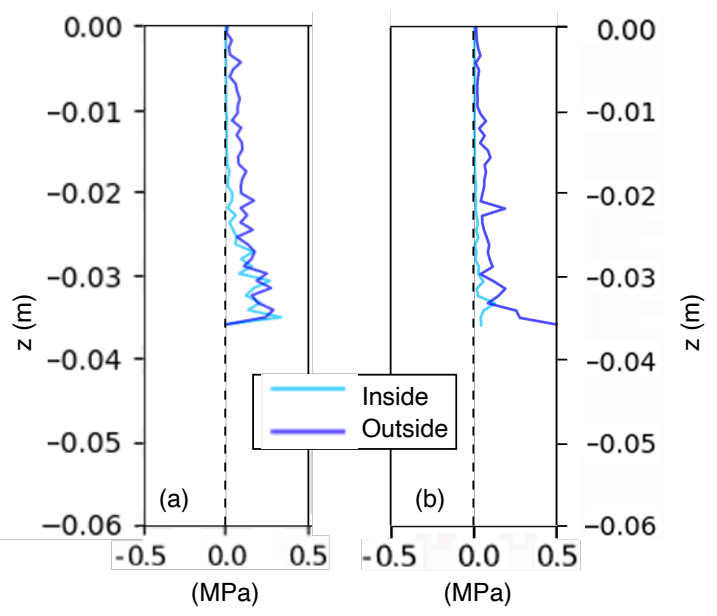

Figure 7. Horizontally-averaged $\sigma_{r r}$ stress acting on the inner (light blue) and outer (dark blue) sides of the pile shaft: (a) $F R$ sample, (b) $+R$ sample.

\section{Conclusions}

In the DEM model used for this study, the ratio of the pile diameter to the mean particle size is 10 , which means that the model clearly does not reproduce field conditions. However, the model proves to reproduce a number of features associated with the installation of open-ended pipe piles, and provides interesting information at the particle scale.

Close to the pile tip, the granular assembly experiences volume expansion - as already observed with $\mathrm{x}$-ray tomography on small-scale laboratory experiments. This is a distinct difference between hollow, open-ended piles and closed-ended piles, for which volume compaction is rather observed close to the pile tip (e.g., the x-ray tomographic observations by [15]). The stress induced in the granular assembly by the penetration of the pile strongly depend on whether there is or not rolling resistance at the particleto-particle contact. Rolling resistance makes the particles close to the pile tip act like a stiff cap, which increases the resistance against pile penetration, and reduces the interaction between the pile shaft and the particles on both the inner and outer sides of the shaft.

The next obvious step of this research will include the comparison of the numerical results with those that can be obtained from small-scale experiments on a mini calibration chamber, where the mechanisms occurring at the grain scale will be analysed quantitatively using x-ray tomography and three-dimensional digital image correlation, similar to what was done in [2] for closed-ended piles.

\section{References}

[1] M. Silva, G. Combe, P. Foray, F. Flin, B. Lesaffre, AIP Conference Proceedings 1542, 297 (2013)

[2] J. Doreau-Malioche, G. Combe, G. Viggiani, J.B. Toni, Geotech Lett 8, 66 (2018)

[3] J. Doreau-Malioche, A. Galvis-Castro, R. TovarValencia, G. Viggiani, G. Combe, M. Prezzi, R. Salgado, Geotech Lett 9, 254 (2019)

[4] J. Doreau-Malioche, G. Combe, J. Toni, G. Viggiani, EPJ Web Conf. 140, 07014 (2017)

[5] P.A. Cundall, O.D.L. Strack, Géotechnique 29, 47 (1979)

[6] F. Radjaï, F. Dubois, Discrete-element modeling of granular materials. (ISTE, London, 2011)

[7] B. Saint-Cyr, K. Szarf, C. Voivret, E. Azema, V. Richefeu, J.Y. Delenne, G. Combe, C. NouguierLehon, P. Villard, P. Sornay et al., EPL 98, 44008 (2012)

[8] E.M. Charalampidou, G. Combe, G. Viggiani, J. Lanier, Mechanical behavior of mixtures of circular and rectangular 2D particles, in Powders and grains 2009, edited by Nakagawa, M and Luding, S (2009), Vol. 1145 of AIP Conference Proceedings, pp. 821-824

[9] N. Estrada, E. Azéma, F. Radjai, A. Taboada, Phys. Rev. E 84, 011306 (2011)

[10] Y. Kikuchi, T. Sato, T. Mizutani, Y. Morikawa, Plugging Mechanism of Open-Ended Piles. (2013), pp. 406-413

[11] C.F.M. Magalhães, A.P. Atman, G. Combe, J.G. Moreira, Pap. Phys. 6, 060007 (2014)

[12] J. Weber, Bul. liaison P. et Ch. 20, 3.1 (1966)

[13] D.J. White, M.D. Bolton, Proceedings of the Institution of Civil Engineers - Geotechnical Engineering 158, 3 (2005)

[14] R. Jardine, B. Zhu, P. Foray, Z. Yang, Géotechnique 63, 1 (2013)

[15] P. Paniagua, E. Andò, M. Silva, A. Emdal, S. Nordal, G. Viggiani, Geotech Lett 3, 185 (2013) 